\title{
Multimodal imaging of a rare type of giant aortic arch aneurysm with malformation of the aortic arch
}

Da Zhu, MD, and Zhong Wu, MD

A symptom-free 35-year-old female patient with a giant mass in the upper chest diagnosed on routine chest radiography was seen at our hospital. Transthoracic

From the Department of Cardiovascular Surgery, West China Hospital, Sichuan University, Sichuan, China.

Disclosures: Authors have nothing to disclose with regard to commercial support. Received for publication March 24, 2014; accepted for publication March 26, 2014 available ahead of print April 24, 2014.

Address for reprints: Zhong Wu, MD, Department of Cardiovascular Surgery, West China Hospital, Chengdu, Sichuan, PR China 610041 (E-mail: wuzhong71@163.com).

J Thorac Cardiovasc Surg 2014;148:3238-9

$0022-5223 / \$ 36.00$

Copyright (c) 2014 by The American Association for Thoracic Surgery

http://dx.doi.org/10.1016/j.jtcvs.2014.03.048 echocardiographic imaging revealed an aneurysm formation in the aortic arch without intracardiac abnormality. Further computed tomographic as well as magnetic resonance imaging angiographic examinations were then used for more detailed evaluation (Figure 1). In contrast to commonly seen aortic arch aneurysms, this giant aortic aneurysm originated from a malformed aortic arch. As shown in Figure 1, the ascending aorta was directly connected into this giant aneurysm at the level distal to the bifurcation of left internal carotid artery, whereas the descending aorta was derived from another portion of the aneurysm proximal to the entrance port of the ascending aorta, forming a unique loop structure of vascular trace.

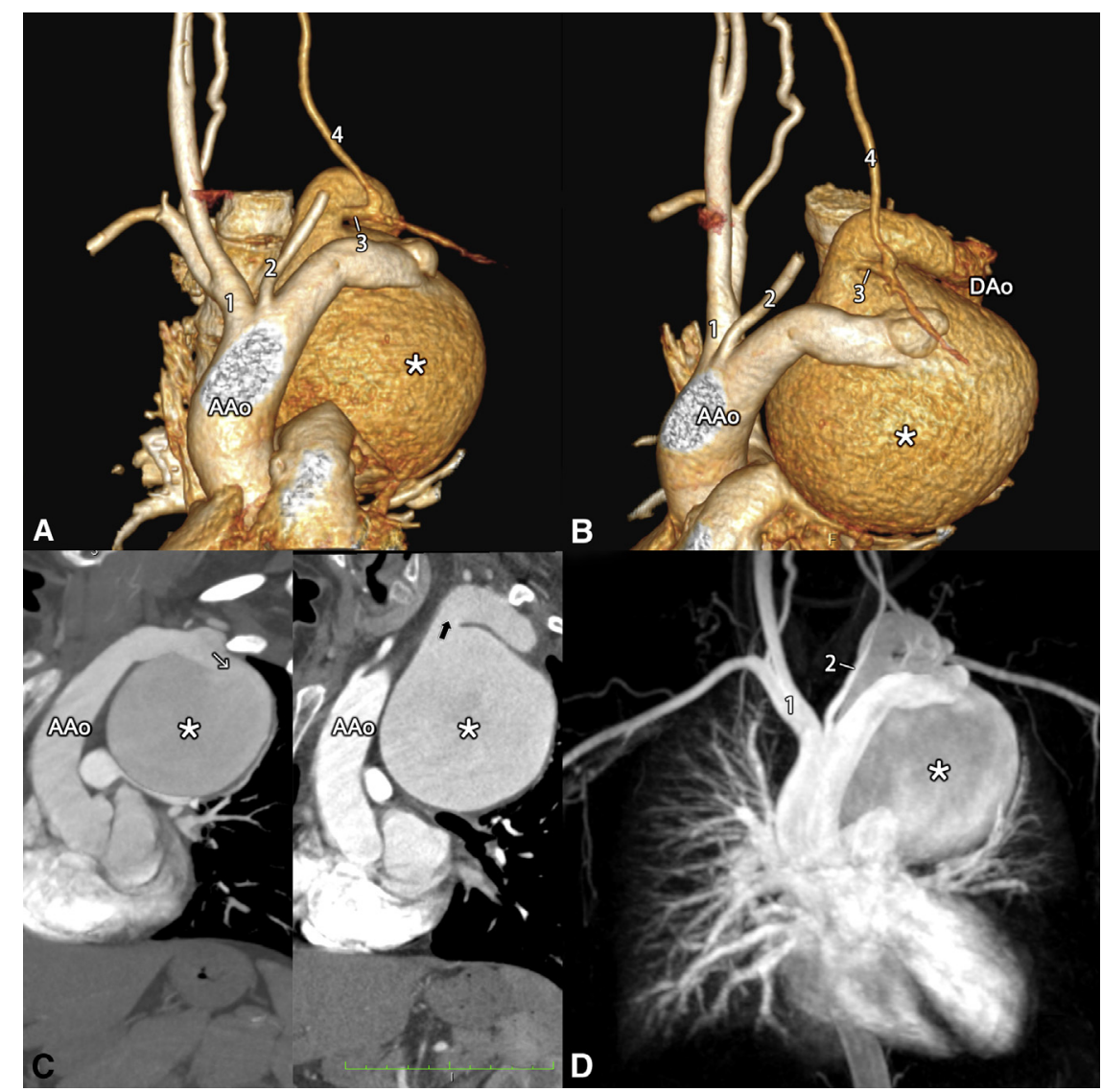

FIGURE 1. Multimodal imaging of an aortic arch aneurysm with malformation of the aortic arch. A and B, Three-dimensional computed tomographic angiographic imaging shows the giant aortic arch aneurysm (asterisk). A mismatch of the entrance and the exit ports of the aneurysm form a unique loop structure of vascular trace. Both the right and left internal carotid arteries are derived from the ascending aorta proximal to the aneurysm, whereas the left subclavian artery originates from the aorta distal to the aneurysm. C, Two-dimensional computed tomographic imaging shows the entrance as well as exit ports of the giant aneurysm. The white arrow indicates the entrance port of the aneurysm; the black arrow indicates the exit port of the aneurysm. $\mathrm{D}$, Magnetic resonance imaging angiographic image also confirms a loop structural malformation of the aortic arch with giant aneurysm formation. 4 , Left vertebral artery; 3 , left subclavian artery; 2 , left internal carotid artery; 1 , right brachiocephalic artery; AAo, ascending aorta; $D A o$, descending aorta. 

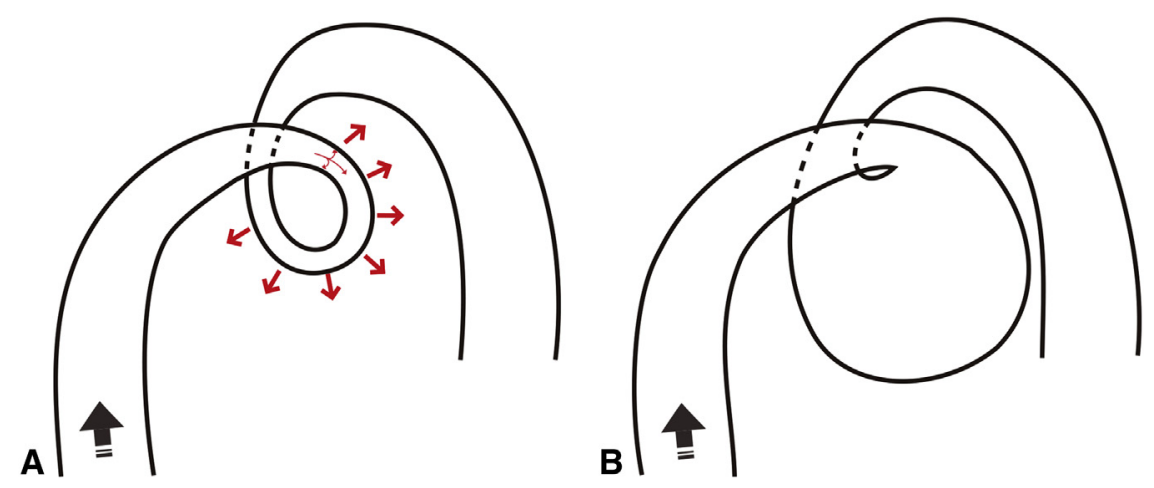

FIGURE 2. Schematic view of suspected etiology of aortic malformation and subsequent aneurysm formation as a result of unique hemodynamic character. A loop malformation of aortic arch would cause the turbulence flow and increase the shear stress to the vascular wall (A), which then would subsequently cause the dilation of the vessel wall as well as the aneurysm (B). Black arrow, direction of the blood flow in aorta. Red arrow, direction of the shear stress from turbulent blood flow.

The left subclavian artery originated from the aortic arch distal to the aneurysm. We speculated that this condition was due the previous malformation of the aortic arch, a native vascular loop anomaly of the aortic arch, with the unique hemodynamic character of this vascular loop curvature then leading to the formation (Figure 2). This unique anatomy precluded a minimally invasive interventional procedure and posed a great challenge for traditional open thoracic surgery. Multimodal imaging methods played an important role in the preoperative evaluation. 